\title{
DIAGRAM VECTORS AND TIGHT FRAME SCALING IN FINITE DIMENSIONS
}

\author{
Martin S. Copenhaver, Yeon Hyang Kim, Cortney Logan, Kyanne \\ Mayfield, Sivaram K. Narayan, Matthew J. Petro and Jonathan \\ SHEPERD
}

Abstract. We consider frames in a finite-dimensional Hilbert space $\mathscr{H}_{n}$ where frames are exactly the spanning sets of the vector space. The diagram vector of a vector in $\mathbb{R}^{2}$ was previously defined using polar coordinates and was used to characterize tight frames in $\mathbb{R}^{2}$ in a geometric fashion. Reformulating the definition of a diagram vector in $\mathbb{R}^{2}$ we provide a natural extension of this notion to $\mathbb{R}^{n}$ and $\mathbb{C}^{n}$. Using the diagram vectors we give a characterization of tight frames in $\mathbb{R}^{n}$ or $\mathbb{C}^{n}$. Further we provide a characterization of when a unit-norm frame in $\mathbb{R}^{n}$ or $\mathbb{C}^{n}$ can be scaled to a tight frame. This classification allows us to determine all scaling coefficients that make a unit-norm frame into a tight frame.

Mathematics subject classification (2010): 42C15, 05B20, 15A03.

Keywords and phrases: Frames, tight frames, tight frame scaling, diagram vectors, Gramian operator.

\section{REFERENCES}

[1] J. J. Benedetto And M. Fickus, Finite Normalized Tight Frames, Adv. Comput. Math., (2003), 18, p. 357-385.

[2] P. G. Casazza And N. Leonhard, Classes of finite equal norm Parseval frames, Frames and operator theory in analysis and signal processing, Contemp. Math., Vol. 451, p. 11-31, Amer. Math. Soc., Providence, RI (2008).

[3] P. G. Casazza, M. Fickus, J. KovaČević, M. T. Leon and J. C. Tremain, A Physical Interpretation of Finite Frames, Appl. Numer. Harmon. Anal., (2006), 2-3, p. 51-76.

[4] P. G. CASAZZA And J. KovaČEviĆ, Equal-Norm Tight Frames with Erasures, Advances in Computational Mathematics, 392, p. 387-430, (2006).

[5] E. W. ChENEY, Introduction to approximation theory, American Mathematical Society, (2000), Providence, Rhode Island.

[6] I. Daubechies, Ten Lectures on Wavelets, SIAM, (1992), Philadelphia, PA.

[7] R. J. Duffin And A. C. Shaeffer, A Class of Nonharmonic Fourier Series, Transactions of the American Mathematical Society, 72, (1952), p. 341-366.

[8] D. Han, K. Kornelson, D. Larson And E. Weber, Frames for undergraduates, Student Mathematical Library, Vol. 40, American Mathematical Society, Providence, RI, (2007), p. xiv+295.

[9] R. A. Horn AND C. R. Johnson, Matrix Analysis, Cambridge University Press, (1985).

[10] K. V. Mital, Optimization Methods in Operations Research and System Analysis, New Age International, (2007).

[11] A. Ron AND Z. Shen, Frames and stable bases for shift-invariant subspaces of $L_{2}\left(\mathbf{R}^{d}\right)$, Canadian Journal of Mathematics (Journal Canadien de Mathématiques), Vol. 47, (1995), 5, p. 1051-1094.

[12] F. Rosenblatt, The perceptron: A theory of statistical separability in cognitive systems, Cornell Aeronautical Laboratory, Inc., Rep. No. VG-1196-G-1. U.S. Department of Commerce, Office of Technical Services, PB 151247, (1958), p. xii+262.

[13] N. SoheILI AND J. PENA, A smooth perceptron algorithm, (2011). 